though tricksters, may yet be mentally, and sometimes physically, ill; and that we who are not can, as well as being clever, also be kind.

How can cases of Munchausen syndrome be recognised early in the consultation? The clinician's suspicion remains all important.

We thank Mr R Touquet and Dr T Challoner for their advice and assistance in the production of this case report. The tracking of the case by Pamela Dymek is gratefully acknowledged.
1 Asher R. Munchausen's syndrome. Lancet 1951;i:339-41.

2 Birch CA. Munchausen's syndrome. Lancet 1951;i:412.

3 Banajee AK. Trauma and Munchausen's syndrome. Arch Emerg Med 1991;8:217-8.

4 Cottam SN, Cuthbert AC, Parapia LA. Munchausen AIDS and haemophilia. Eur $\mathcal{F}$ Haematol 1991;4b:125.

5 Ayass M, Bussing R, Mehta P. Munchausen's syndrome presenting as haemophilia. Paediatr Haematol Oncol 1993; presenting as

6 Mcdonald J, Wafer K. Munchausen's syndrome masquerading as AIDS induced depression. $\mathrm{Br} \quad \mathcal{F}$ Psychiatry 1989;154:420-1.

7 Jones JR, Horrocks FA. Fictitious epilepsy associated with amnesia. Br F Psychiatry 1987;150:257-8.

\title{
Oesophageal perforation: a rare complication of minor blunt trauma
}

\author{
Poole Hospital NHS \\ Trust, \\ Longfleet Road, \\ Poole, \\ Dorset BH15 2JB, \\ United Kingdom \\ G L A Cumberbatch \\ M Reichl \\ Correspondence to: \\ G Cumberbatch, Registrar in \\ A\&E Medicine \\ address. \\ Accepted for publication \\ 22 November 1995
}

\section{G L A Cumberbatch, M Reichl}

\begin{abstract}
Oesophageal perforation following blunt trauma is rare and accounts for less than $10 \%$ of all oesophageal ruptures. Review of published reports revealed only two cases of isolated oesophageal perforation after minor blunt trauma, and these were as a direct result of the Heimlich manoeuvre.
\end{abstract}

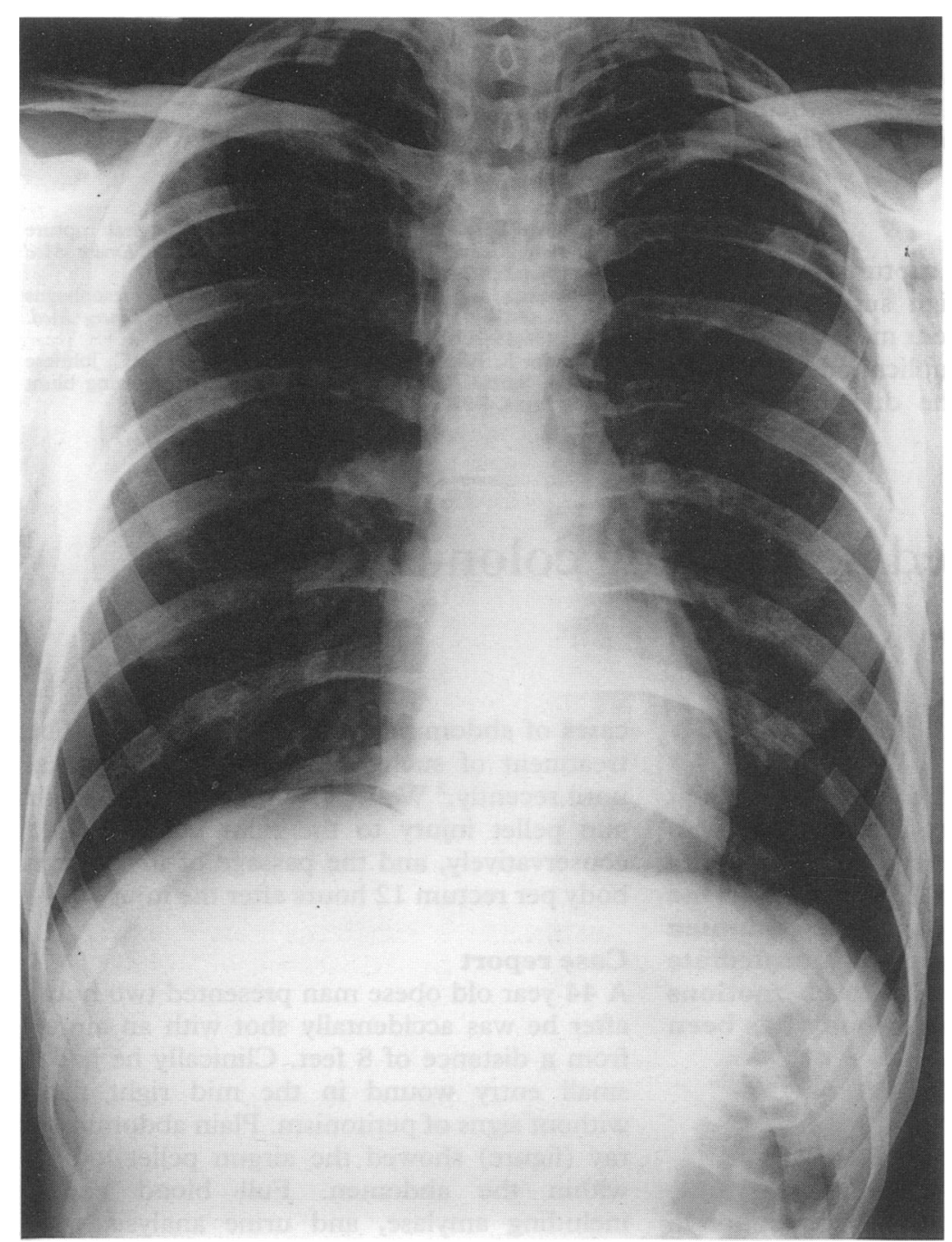

Figure 1 Erect chest $x$ ray with subcutaneous air and a pneumomediastinum.
This paper describes a case of perforation of the oesophagus as an isolated injury following blunt minor trauma.

( f Accid Emerg Med 1996;13:295-296)

Key terms: minor blunt trauma; oesophageal perforation.

Review of published reports revealed only two cases of isolated oesophageal perforation after minor blunt trauma, and these were as a direct result of the Heimlich manoeuvre. ${ }^{12}$ We present a case of perforation of the oesophagus as an isolated injury following blunt minor trauma.

\section{Case report}

A 26 year old man presented to our accident and emergency ( $A \& E$ ) department complaining of neck swelling and dyspnoea, following a blow to the interscapular region of his back during a rugby match. He initially felt "winded" and immediately stopped playing, arranging his own transport to the hospital. On arrival he also complained of dysphagia and a change in his voice.

He had no significant past medical history. On examination he was apyrexial, had a pulse rate of $62 / \mathrm{min}$, a respiratory rate of $24 / \mathrm{min}$, a blood pressure of $139 / 65 \mathrm{~mm} \mathrm{Hg}$, and oxygen saturation of $99 \%$ on air. $\mathrm{He}$ had obvious surgical emphysema extending from the root of his neck down to the nipple line bilaterally. The rest of the physical examination was unremarkable.

Chest radiography (fig 1) revealed marked subcutaneous surgical emphysema, a pneumomediastinum, and a very small apical pneumothorax. Lateral cervical spine $x$ ray (fig 2) showed a large volume of air in the prevertebral space, with anterior displacement of the prevertebral fascia.

A clinical diagnosis of ruptured oesophagus was made. Iohexol (Omnipaque) swallow was arranged urgently and confirmed a perforation in the upper one third of the oesophagus.

He was admitted to the surgical ward and treated conservatively, with intravenous anti- 


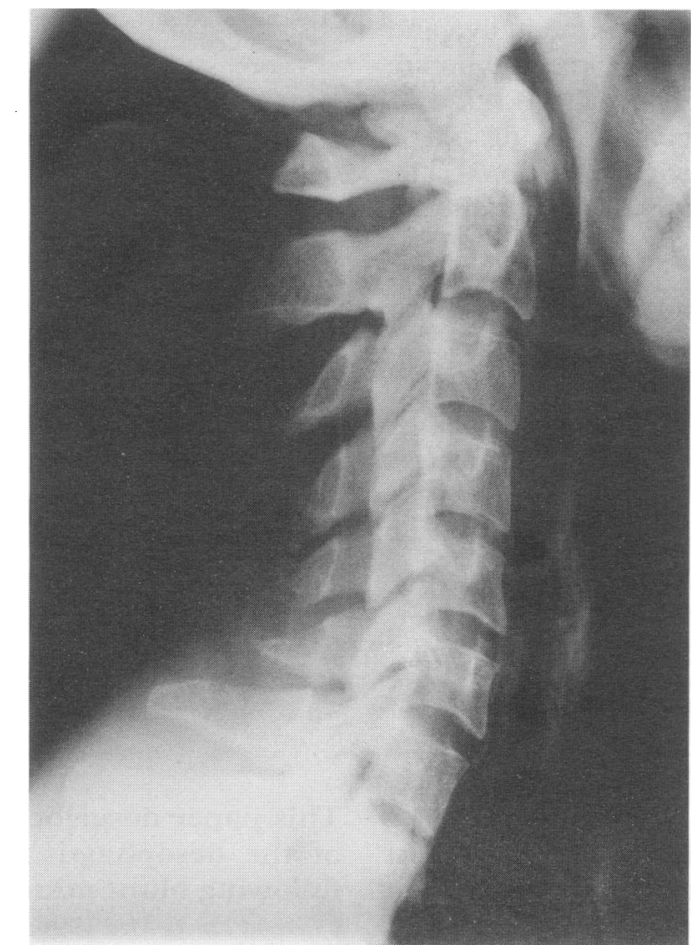

Figure 2 Lateral radiograph of the neck with significant prevertebral air extending to the skull base.

biotics and enteral feeding through a fine bore feeding tube which was inserted under $x$ ray guidance.

The patient made an uneventful recovery and was discharged after 10 days, when he was able to tolerate solids orally. No follow up was thought necessary.

\section{Discussion}

The clinical diagnosis of ruptured oesophagus is always difficult. A high suspicion of this injury is essential to prevent missed or delayed diagnosis, as its complications carry a significant mortality. The diagnosis is even more difficult following blunt trauma, as often the presenting features are those of the associated injuries, for example pneumothorax, haemothorax, or flail chest.

A previous search of published reports on perforation of the oesophagus by blunt external trauma $^{3}$ reviewed 63 cases and detailed the mechanism of injury, associated injuries, clinical findings, and outcome. In all but two cases the mechanism of injury was significant trauma, mostly due to road traffic accidents and falls. These two exceptions were cases of trauma from the Heimlich manoeuvre; no cases were caused by minor sports injury.

The study showed that patients had a variety of symptoms, namely dysphagia, dyspnoea, hoarseness, neck pain or swelling, and chest or abdominal pain. Physical signs were absent or appeared only as neck or chest wall surgical emphysema. Radiography of the chest revealed subcutaneous air, pneumomediastinum or pneumothorax, while lateral cervical spine $x$ ray showed air posterior to the prevertebral fascia.

Our patient had most of these clinical features and hence the diagnosis was made relatively easily. However, if surgical emphysema had been absent or not noticed the diagnosis would have been easily missed.

This case shows the importance of suspecting a ruptured oesophagus when there are suggestive clinical features, even in the absence of severe blunt trauma to the chest or abdomen.

We are grateful to Gabbie Valentine for help in the preparation of the manuscript.

1 Haynes DE, Haynes BE, Yong YV. Esophageal rupture complicating Heimlich manoeuvre. Am 7 Emerg Med 1984;2:507-9.

2 Meredith MJ, Liebowitz R. Rupture of the oesophagus caused by the Heimlich manoeuvre. Ann Emerg Med. 1986;15:106-7.

3 Sandra L, Beal MD, Edward W, Pottmeyer MC, Johnese M, Spisso RN. Esophageal perforation following blunt trauma. F Trauma 1988;28:1425-32.
Department of Surgery, The Royal Gwent Hospital, Newport, Gwent NP9 2UB United Kingdom T O Oshodi D Bowrey

Correspondence to: T O Oshodi, 122 Cholmley Gardens, Fortune Green Road, West Hampstead, London NW6 1AA

Accepted for publication 15 November 1995

\section{Uncomplicated penetrating colonic injury}

\section{T O Oshodi, D Bowrey}

\begin{abstract}
The case of a patient with an air gun pellet injury to the right colon is reported. This was treated conservatively, and the pellet was passed per rectum 12 hours after the injury. Gunshot wounds to the abdomen do not necessarily warrant immediate laparotomy. Sieving of bowel motions may identify if the foreign body has been passed.

( $(\mathcal{A}$ Accid Emerg Med 1996;13:296-297)
\end{abstract}

Key terms: gunshot wound; colon penetration

Gunshot abdominal injuries are uncommon in the United Kingdom but account for $47 \%$ of cases of abdominal trauma in the USA. ${ }^{1}$ The treatment of such injuries was controversial until recently. ${ }^{2}$ We report a patient with an air gun pellet injury to the right colon treated conservatively, and the passage of the foreign body per rectum 12 hours after the injury.

\section{Case report}

A 44 year old obese man presented two hours after he was accidentally shot with an airgun from a distance of 8 feet. Clinically he had a small entry wound in the mid right flank without signs of peritonism. Plain abdominal $x$ ray (figure) showed the airgun pellet lodged within the abdomen. Full blood count, including amylase, and urine analysis were normal. He was placed on antibiotics and 